\title{
Scattering-based hole burning mediated by localized surface plasmon resonance in photoreactive random media containing Ag nanoparticles
}

\section{$\operatorname{AUTHOR}(\mathrm{S})$ :}

Murai, Shunsuke; Fujimoto, Yutaka; Iwata, Koji; Fujita, Koji; Tanaka, Katsuhisa

\section{CITATION:}

Murai, Shunsuke ... [et al]. Scattering-based hole burning mediated by localized surface plasmon resonance in photoreactive random media containing Ag nanoparticles. Applied Physics Letters 2011, 98(12): 121917.

ISSUE DATE:

2011-03

URL:

http://hdl.handle.net/2433/159437

RIGHT:

(C) 2011 American Institute of Physics 


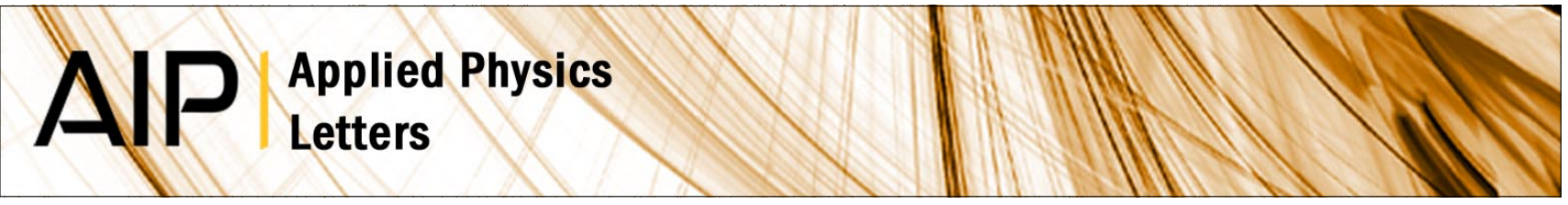

\section{Scattering-based hole burning mediated by localized surface plasmon resonance in photoreactive random media containing Ag nanoparticles}

Shunsuke Murai, Yutaka Fujimoto, Koji Iwata, Koji Fujita, and Katsuhisa Tanaka

Citation: Appl. Phys. Lett. 98, 121917 (2011); doi: 10.1063/1.3567929

View online: http://dx.doi.org/10.1063/1.3567929

View Table of Contents: http://apl.aip.org/resource/1/APPLAB/v98/i12

Published by the American Institute of Physics.

\section{Related Articles}

Two-photon spectral hole burning spectroscopy of InAs/GaAs quantum dots Appl. Phys. Lett. 97, 011903 (2010)

Analytical formulas for low-fluence non-line-narrowed hole-burned spectra in an excitonically coupled dimer J. Chem. Phys. 131, 234104 (2009)

Scattering-based hole burning through volume speckles in a random medium with tunable diffusion constant Appl. Phys. Lett. 93, 151912 (2008)

2-methylterrylene in hexadecane: Do we see single rotational quantum jumps of methyl groups?

J. Chem. Phys. 128, 044508 (2008)

\section{Additional information on Appl. Phys. Lett.}

Journal Homepage: http://apl.aip.org/

Journal Information: http://apl.aip.org/about/about_the_journal

Top downloads: http://apl.aip.org/features/most_downloaded

Information for Authors: http://apl.aip.org/authors

\section{ADVERTISEMENT}
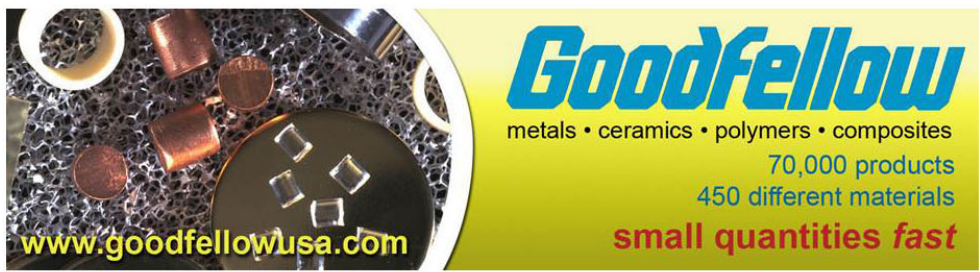


\title{
Scattering-based hole burning mediated by localized surface plasmon resonance in photoreactive random media containing Ag nanoparticles
}

\author{
Shunsuke Murai, ${ }^{1, a)}$ Yutaka Fujimoto, ${ }^{1}$ Koji Iwata, ${ }^{1}$ Koji Fujita,,${ }^{1,2}$ and Katsuhisa Tanaka ${ }^{1}$ \\ ${ }^{1}$ Department of Material Chemistry, Graduate School of Engineering, Kyoto University, Katsura, \\ Nishikyo-ku, Kyoto 615-8510, Japan \\ ${ }^{2}$ PRESTO, Japan Science and Technology Agency (JST), 4-1-8 Honcho, Kawaguchi, \\ Saitama 332-0012, Japan
}

(Received 8 December 2010; accepted 13 February 2011; published online 25 March 2011)

\begin{abstract}
Photoreactive random media containing silver nanoparticles (Ag NPs) have been fabricated, and hole burning effects based on the interference of multiply scattered light have been investigated. Through the analysis of hole profiles, transport mean free path $\ell$ is estimated for the light with the wavelength of $585 \mathrm{~nm}$. We find that $\ell$ in the samples embedded with Ag NPs is much shorter compared with that in a medium containing the same amount of titania $\left(\mathrm{TiO}_{2}\right) \mathrm{NPs}$, indicating that Ag NPs scatter $585 \mathrm{~nm}$ lightwaves much stronger than $\mathrm{TiO}_{2}$ NPs because of their larger scattering cross sections due to the localized surface plasmon resonance. (C) 2011 American Institute of Physics. [doi:10.1063/1.3567929]
\end{abstract}

Random media refer to complex materials in which scatterers are randomly arranged on an optical length scale. Multiple scattering and interference in a random medium bring about interesting phenomena such as coherent backscattering (CBS) ${ }^{1-3}$ random lasers, ${ }^{4,5}$ and scattering-based hole burning effect. ${ }^{6-8}$ Among them, scattering-based hole burning utilizes the high sensitivity of an interference pattern to small changes in the source frequency, polarization, and wave vector. ${ }^{9,10}$ Memories can be imprinted by irradiating a random medium containing photochromic species with a monochromatic laser that preferentially photobleaches the species located in the interference pattern.

Thus far, in many experimental studies on random media, nanoparticles (NPs) were adopted as the scatterers. Assuming that all the NPs in the medium are identical and every scattering event is independent of other scattering events, transport mean free path $\ell$ is expressed in terms of the transport cross section $\sigma_{\text {tra }}$ of an NP and the number density of NPs $\rho$ as $\ell=1 / \rho \sigma_{\text {tra }}$, where $\sigma_{\text {tra }}$ can be expressed in terms of scattering $\left(\sigma_{\mathrm{sca}}\right)$ and absorption $\left(\sigma_{\mathrm{abs}}\right)$ cross sections and an asymmetric factor $g:(1-g) \sigma_{\mathrm{sca}}+\sigma_{\mathrm{abb}}{ }^{11} \ell$ is related to the diffusion constant $D$ as $D=v_{\mathrm{E}} \ell / 3$, where $v_{\mathrm{E}}$ is the energy velocity of light. ${ }^{12}$ For fabricating a stronglyscattering medium, dielectric NPs having a high refractive index, such as titania $\left(\mathrm{TiO}_{2}\right)$, are commonly employed to attain a large $\sigma_{\text {sca }}$. Recently, the use of metal NPs as scatterers has been studied; metal NPs potentially possess excellent scattering properties due to the localized surface plasmon resonance (LSPR). $\sigma_{\text {sca }}$ of a metal NP is much larger than that of a dielectric NP. For example, according to the Mie theory, a silver (Ag) NP (diameter $=100 \mathrm{~nm}$, dielectric constants were obtained from literature ${ }^{13}$ ) in a matrix with a refractive index of 1.49 exhibits LSPR at a wavelength of approximately $580 \mathrm{~nm} ; \sigma_{\text {sca }}$ at this value is $4 \times 10^{-14} \mathrm{~m}^{2}$. $\sigma_{\text {sca }}$ of a $\mathrm{TiO}_{2} \mathrm{NP}$ (refractive index $=2.7$ ) under the same conditions is $2 \times 10^{-15} \mathrm{~m}^{2}$, i.e., 1/20th of that of the Ag NP. Recent experimental reports stated that random lasers containing metal NPs exhibit a lower laser threshold than those

${ }^{a)}$ Electronic mail: murai@dipole7.kuic.kyoto-u.ac.jp. containing dielectric NPs; $;{ }^{14-16}$ this observation suggests that metal NPs are a better choice.

In the present study, we have prepared photoreactive random media containing Ag NPs with a diameter of approximately $100 \mathrm{~nm}$ and examined the scattering-based hole burning effect. We calculate $\ell$ by analyzing the hole profile and compare $\ell$ with that obtained in the case of media embedded with $\mathrm{TiO}_{2} \mathrm{NPs}$. The value of $\ell$ is smaller in the case of Ag-containing media, which points to a larger $\sigma_{\text {tra }}$ of Ag NP.

Ag NPs were synthesized by a two-step seed-mediated growth method. First, a $0.188 \mathrm{M}$ silver nitrate aqueous solution $\left[\mathrm{AgNO}_{3}(\mathrm{aq}), 3.0 \mathrm{ml}\right]$ was added to water $(8.0 \mathrm{ml})$ in the presence of poly(vinyl pyrrolidone) (molecular weight: $55000 ; 1.41 \mathrm{~g}$ ) and stirred for $24 \mathrm{~h}$ at $60^{\circ} \mathrm{C}$ to obtain a seed solution. Next, ethylene glycol (EG, $4.0 \mathrm{ml}$ ) was added to the seed solution $(1.5 \mathrm{ml})$ and it was heated for $20 \mathrm{~h}$ at $120{ }^{\circ} \mathrm{C}$ while being continuously stirred. Then, EG $(2.0 \mathrm{ml})$ and $0.188 \mathrm{M} \mathrm{AgNO}_{3}$ (aq) $(2.0 \mathrm{ml})$ were added to the solution, and the mixture was stirred for $6 \mathrm{~h}$ at $120^{\circ} \mathrm{C}$ to increase the size of the NPs up to $100 \mathrm{~nm}$. The obtained NPs were coated with an anionic polymer, poly(4-styrenesulfonic acid) (PSS; molecular weight: 75000 ), for obtaining better dispersion by mixing the suspension of Ag NPs $(3.0 \mathrm{ml})$ with $20 \mathrm{ml}$ of a 1 wt $\%$ PSS aqueous solution under sonication.

The photoreactive films were prepared by adding photochromic 1,3,3-trimethylindolino-6' ${ }^{\prime}$-nitrobenzopyrylospiran (SP1) and the solution of PSS-coated Ag NPs to a tetrahydrofuran solution of poly(methyl methacrylate) (PMMA; molecular weight: 120000 ). The solvent evaporated slowly in air at $60{ }^{\circ} \mathrm{C}$, forming films with a thickness of several hundred micrometers and containing $6.3 \times 10^{-2} \mathrm{M}$ SP1 in the PMMA matrix. We prepared various films having different $\mathrm{Ag}$ contents and named them as Ag- $x$ ( $x$ $=0,1,3,8,12,20)$, where $x$ was the relative value to $\rho$ of $\mathrm{Ag}-1$, for which $\rho=7.4 \times 10^{16} \mathrm{~m}^{-3}$. For comparison, photoreactive media containing $\mathrm{TiO}_{2} \mathrm{NPs}$ (diameter $=100 \mathrm{~nm}$, PT501A, Ishihara Sangyo) instead of Ag NPs were also prepared and named as $\mathrm{TiO}_{2}-y \quad(y=1,3,8,12$, $20,50,100,200)$. It should be noted here that $\mathrm{TiO}_{2}-1$ and Ag-1 contain the same number of NPs. 

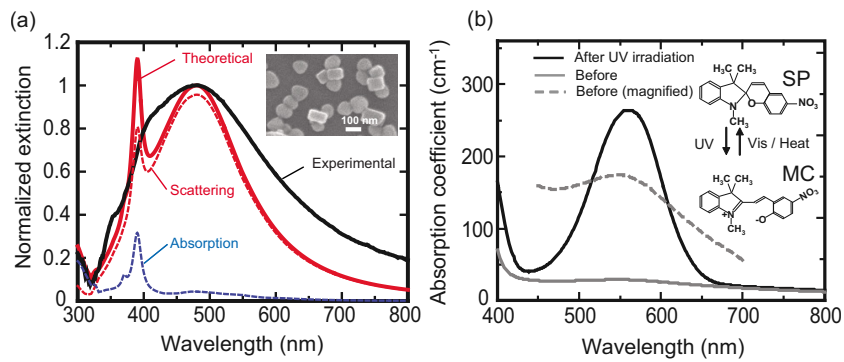

FIG. 1. (Color online) (a) Normalized extinction spectrum for Ag NPs dispersed in ethanol (solid curve). Also shown are the theoretical spectra for an $\mathrm{Ag}$ sphere in ethanol calculated using Mie theory. Theoretical extinction is represented by a solid curve, which consists of scattering and absorption components as indicated by dashed curves. The inset shows a SEM image for Ag NPs dispersed onto a silica glass substrate. (b) Optical absorption spectra for Ag-1 sample before (gray solid curve) and after (black) UV light irradiation $(\lambda=365 \mathrm{~nm})$. For clarity, the spectrum before irradiation is magnified and shown as gray broken curve in a wavelength range of 450-700 $\mathrm{nm}$. The inset illustrates two different forms of SP1; SP and MC.

The extinction spectrum of the Ag NPs dispersed in ethanol is shown in Fig. 1(a). The extinction peak at around 480 $\mathrm{nm}$ and the broad shoulder at around $390 \mathrm{~nm}$ can be ascribed to the dipole and quadrupole modes of the LSPR of the Ag NPs, respectively. Moreover, the figure shows a theoretical extinction spectrum calculated on the basis of the Mie theory for an $\mathrm{Ag}$ sphere in ethanol having a diameter of $96 \mathrm{~nm}$. The theoretical peak position of the dipole mode is in good agreement with that of the experimental spectrum. The theoretical extinction curve consists of scattering and absorption components, as shown by the dashed curves. The scattering component is much larger than the absorption component. The broader experimental curve indicates a variation in the diameter and shape of the fabricated NPs. Moreover, the size of the Ag NPs is confirmed by scanning electron microscopy [inset of Fig. 1(a)].

Figure 1(b) shows the typical absorption spectra of the film (Ag-1). An as-prepared (before UV irradiation) film shows an absorption peak at around $550 \mathrm{~nm}$ because of the LSPR of the Ag NPs, while an additional intense absorption peak appears after irradiation with UV light $(\lambda=365 \mathrm{~nm})$. The absorption occurs due to the open-ring merocyanine (MC) form of the dye; UV light transforms the structure of SP1 from closed-ring spiropyran (SP) to MC [inset in Fig. $1(\mathrm{~b})] .{ }^{17}$ While the SP form of the dye hardly shows fluorescence, the MC form is fluorescent, with the maximum emission being observed at around $660 \mathrm{~nm}$ when excited by visible light; moreover, the MC form can be photobleached into SP form when the intensity of the visible light is high. From the data at a wavelength of $585 \mathrm{~nm}$, the absorption length $\ell_{\mathrm{a}}$ is estimated to be around $1000 \mu \mathrm{m}$ for Ag-0 before the UV irradiation. This indicates that $\ell_{\mathrm{a}}$ of $\mathrm{TiO}_{2}-y$ before the UV irradiation (with dyes in SP form) is of the order of $1000 \mu \mathrm{m}$ and that of $\operatorname{Ag}-x(x \neq 0)$ is smaller than $1000 \mu \mathrm{m}$ due to the absorption by Ag NPs.

Scattering-based hole burning was evaluated by using a dye laser (rhodamine 6G) pumped by an $\mathrm{Ar}^{+}$laser (Innova 300, Coherent, USA); the dye laser was used as both the writing and reading beams. First, the film was placed on a rotatable stage and irradiated with UV light for $15 \mathrm{~min}$ to convert the SP1 molecules to the MC form. Second, we illuminated the sample with a writing beam obtained from the cw dye laser $\left(1 \mathrm{~mW}\right.$; wavelength $\lambda_{\mathrm{w}}=585 \mathrm{~nm}$; wave vector

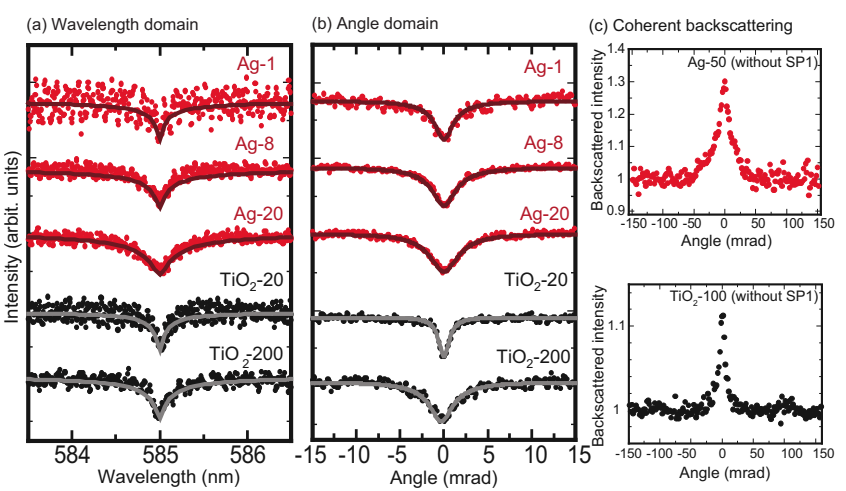

FIG. 2. (Color online) (a) Dependence of emission intensity on excitation wavelength of reading beam for representative samples. The holes were burned using a writing laser beam the wavelength of which was $\lambda_{w}$ $=585 \mathrm{~nm}$. (b) Dependence of emission intensity on incident angle of reading beam. The holes were burned at $0 \mathrm{rad}$ under irradiation with a writing laser beam $\left(\lambda_{\mathrm{w}}=585 \mathrm{~nm}\right)$. Closed circles denote experimental data and solid curves represent theoretical ones based on diffusion approximation [Eq. (1)]. (c) The backscattered intensities normalized to the diffuse background for Ag-50 without SP1 (top figure) and $\mathrm{TiO}_{2}-100$ without SP1 (bottom).

$k_{\mathrm{w}}=2 \pi \sin \theta / \lambda$, where $\theta$ is the incident angle) for about $30 \mathrm{~s}$. This illumination reconverts the MC forms to the SP forms in the interference pattern specific to $\lambda_{\mathrm{w}}$ (frequency $\omega_{\mathrm{w}}$ ) and $k_{\mathrm{w}}$. In the reading process, the laser beam was attenuated by a factor of $200(5 \mu \mathrm{W})$ and used as the reading beam to probe the hole without causing any additional photoreactions. The spot that was irradiated with the writing beam prior to reading was excited by a reading beam having wavelength $\lambda_{\mathrm{r}}$ (frequency $\omega_{\mathrm{r}}$ ) and wave vector $k_{\mathrm{r}}$, and the emission intensity was plotted as a function of $\Delta \lambda\left[=\lambda_{\mathrm{r}}-\lambda_{\mathrm{w}}(\Delta \omega\right.$ $\left.\left.=\omega_{\mathrm{r}}-\omega_{\mathrm{w}}\right)\right]$ and $\Delta k\left(=k_{\mathrm{r}}-k_{\mathrm{w}}\right)$.

The representative hole profiles are shown in Fig. 2. All $\mathrm{Ag}-x$ films except $\mathrm{Ag}-0$ exhibit holes in the wavelength and angle domains [Figs. 2(a) and 2(b)]. In contrast, for the $\mathrm{TiO}_{2}-y$ films, no holes are observed in both domains unless $y$ is more than 20 , which indicates that $\ell$ is more than the film thickness when $y=1,3,8$, and 12 . This demonstrates that $\sigma_{\text {tra }}$ is larger for $\mathrm{Ag}$ NPs than for $\mathrm{TiO}_{2} \mathrm{NPs}$. To confirm the larger $\sigma_{\text {tra }}$ of an Ag NP, we conducted a CBS measurement using the $514 \mathrm{~nm}$ line of the $\mathrm{Ar}^{+}$laser ${ }^{18}$ [see Fig. 2(c)]. Ag $\left(\mathrm{TiO}_{2}\right)$-containing films with thicknesses of around $300 \mu \mathrm{m}$ and having the same number of NPs as Ag-50 $\left(\mathrm{TiO}_{2}-100\right)$ were prepared without SP1, and they were used as samples. Ag-50 without SP1 (the top figure) shows a wider CBS cone than $\mathrm{TiO}_{2}-100$ without SP1 [the bottom], although the number of Ag NPs contained is half the number of $\mathrm{TiO}_{2} \mathrm{NPs}$. Since a wider CBS cone means a smaller $\ell$, this observation confirms the stronger scattering by Ag NPs. The enhancement in the CBS cones in the backscattering direction (1.3 and 1.1 for $\mathrm{Ag}-50$ without SP1 and $\mathrm{TiO}_{2}-100$ without SP1, respectively) is much smaller than the theoretical value $(=2.0)$. This is primarily due to the limited film thickness; the photons that take paths whose lengths are more than the thickness of the medium are transmitted and do not contribute to the CBS peak. ${ }^{19}$ In the case of the Ag-containing film, the absorption by Ag NPs is an additional reason for the low enhancement. $^{19,20}$

To estimate $\ell$, the obtained hole profiles were analyzed using a theoretical curve based on the diffusion theory. The correlation between the intensities of the fluctuation patterns produced by the writing and reading beams at position $r$ 


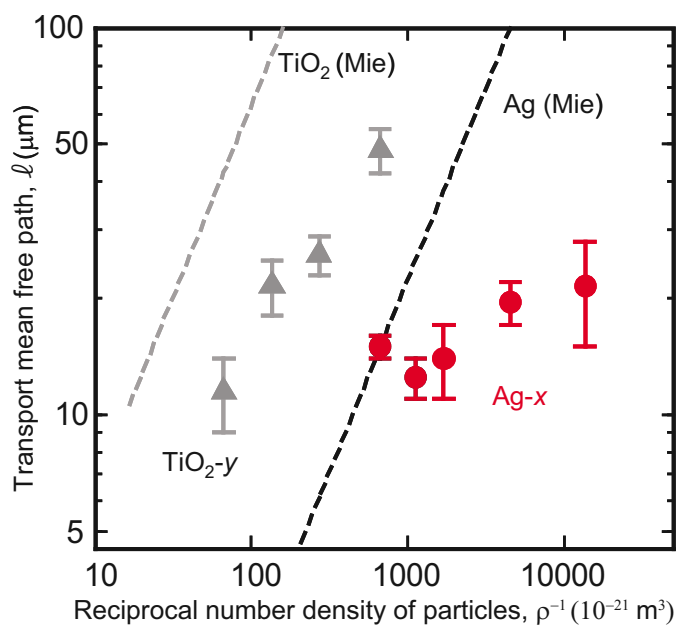

FIG. 3. (Color online) Transport mean free path estimated from the hole profiles as a function of reciprocal number density of NPs for Ag- $x$ (closed circles, $x=1,3,8,12$, and 20) and $\mathrm{TiO}_{2}-y$ (closed triangles, $y=20,50,100$, and 200). Broken lines denote the theoretical relations based on $\ell=1 / \rho \sigma_{\text {tra }}$ for $\mathrm{Ag}-x$ (black) and $\mathrm{TiO}_{2}-y$ (gray).

inside the medium is described by an intensity correlation function $C(\Delta \omega, \Delta k, r),{ }^{7}$ which essentially determines the shape of a hole. For a slab medium occupying a space 0 $<z<L, C(\Delta \omega, \Delta k, z)$ can be represented as

$$
\begin{aligned}
& C(\Delta \omega, \Delta k, z) \\
& \quad=\frac{\cosh [2 \gamma(L+0.7 \ell-z)]-\cos [2 \delta(L+0.7 \ell-z)]}{\cosh [2 \gamma(L+1.4 \ell)]-\cos [2 \delta(L+1.4 \ell)]} .
\end{aligned}
$$

Here $\gamma+i \delta=\left[\Delta k^{2}+\left(c / \ell_{\mathrm{a}}+i \Delta \omega\right) / D\right]^{1 / 2}$. For simplicity, we assumed $v_{\mathrm{E}}=c /\langle n\rangle$, where $\langle n\rangle$ is the average refractive index of the medium and was assumed to be equal to that of the PMMA matrix $(=1.49)$. We fitted the integration of Eq. (1) over $z$ to the experimental hole profiles by considering $\ell_{\mathrm{a}}$ (values in the range of 500-3000 $\mu \mathrm{m}$, expected from extinction spectra) and $\ell$ as fitting parameters. The results are superimposed on the experimental data in Figs. 2(a) and 2(b). The experimental hole profiles can be reproduced well by the theoretical curves.

The values of $\ell$ estimated from the fit are plotted in Fig. 3 as a function of the inverse number density of NPs, $\rho^{-1}$. The error bars reflect the variations in $\ell$ values caused by the various $\ell_{\mathrm{a}}$ values used for the fit. The range of $\ell_{\mathrm{a}}$ in which the hole profile can be reproduced is almost invariant with $y$ for the $\mathrm{TiO}_{2}-y$ series, while the range for the $\mathrm{Ag}-x$ series tends to shift toward smaller $\ell_{\mathrm{a}}$ values upon increasing $x$. For the $\mathrm{TiO}_{2}-y$ samples, $\ell$ decreases monotonically with increasing $\rho$. Compared to the $\mathrm{TiO}_{2}-y$ samples, the $\mathrm{Ag}-x$ series show smaller values of $\ell$ for smaller $\rho$. For example, the value of $\ell$ for Ag-1 (approximately $21 \mu \mathrm{m}$ ) is comparable to that for $\mathrm{TiO}_{2}-100$, suggesting that $\sigma_{\text {tra }}$ of an Ag NP is 100 times larger than that of a $\mathrm{TiO}_{2} \mathrm{NP}$. The theoretical value of $\ell$ estimated from the relation $\ell=1 / \rho \sigma_{\text {tra }}$, in which $\sigma_{\text {tra }}$ was calculated for a sphere (diameter $=100 \mathrm{~nm}$ ) using the Mie theory, is shown by broken lines. The absorption of SP1 was neglected in the calculation since the contribution of absorption estimated in the experiment $\left(\ell_{\mathrm{a}}=500-3000 \mu \mathrm{m}\right)$ was less significant than that of scattering, i.e., $\ell \ll \ell_{\mathrm{a}}$. For both the $\mathrm{Ag}-x$ and $\mathrm{TiO}_{2}-y$ series, the calculated values are more than those estimated from the experimental data. This difference can be ascribed to the fact that NPs used in the experiment had distributions in size and shape. While the slope of the experimentally obtained curve between $\ell$ and $\rho^{-1}$ is similar to the theoretical one for the $\mathrm{TiO}_{2}-y$ films, the slope for the $\mathrm{Ag}-x$ data does not match to the relation $\ell=1 / \rho \sigma_{\text {tra. }}$ A weaker dependence on $\rho^{-1}$ could be due to the effect of multiple scattering. If the scattering is strong, the incident light is screened by other NPs and this results in a lower scattering strength of the system than that estimated when independent scattering is assumed. ${ }^{21}$

In summary, we have prepared photoreactive random media containing Ag NPs as scatterers and experimentally verified that $\sigma_{\text {tra }}$ of $\mathrm{Ag}$ NPs is much larger than that of $\mathrm{TiO}_{2}$ NPs on the basis of scattering-based hole burning effects. The result suggests that Ag NPs can be excellent scatterers with a large $\sigma_{\mathrm{sca}}$ and a reasonable $\sigma_{\mathrm{abs}}$ when their size is appropriate, as expected from the Mie theory.

This study was supported by a Grant-in-Aid for Young Scientists (B) (Grant Nos. 22750188 and 22760512) from the Ministry of Education, Culture, Sports, Science, and Technology (MEXT), Japan.

${ }^{1}$ Y. Kuga and A. Ishimaru, J. Opt. Soc. Am. A 1, 831 (1984).

${ }^{2}$ P. E. Wolf and G. Maret, Phys. Rev. Lett. 55, 2696 (1985).

${ }^{3}$ M. P. Van Albada and A. Lagendijk, Phys. Rev. Lett. 55, 2692 (1985).

${ }^{4}$ D. Wiersma, Nat. Phys. 4, 359 (2008).

${ }^{5}$ H. Cao, J. Phys. A 38, 10497 (2005).

${ }^{6}$ A. Kurita, Y. Kanematsu, M. Watanabe, K. Hirata, and T. Kushida, Phys. Rev. Lett. 83, 1582 (1999).

${ }^{7}$ M. Tomita, T. Ito, and A. Hattori, Phys. Rev. B 64, 180202(R) (2001).

${ }^{8}$ K. Fujita, Y. Ohashi, and K. Hirao, Opt. Lett. 28, 567 (2003).

${ }^{9}$ B. Shapiro, Phys. Rev. Lett. 57, 2168 (1986).

${ }^{10}$ I. Freund, M. Rosenbluh, and S. Feng, Phys. Rev. Lett. 61, 2328 (1988)

${ }^{11}$ A. Ishimaru, Wave Propagation and Scattering in Random Media (Academic, New York, 1978), Vol. 1, Chap. 9, p. 178.

${ }^{12}$ R. Sapienza, P. D. García, J. Bertolotti, M. D. Martín, Á. Blanco, L. Viña, C. López, and D. S. Wiersma, Phys. Rev. Lett. 99, 233902 (2007).

${ }^{13}$ P. B. Johnson and R. W. Christy, Phys. Rev. B 6, 4370 (1972).

${ }^{14}$ G. D. Dice, S. Mujumdar, and A. Y. Elezzabi, Appl. Phys. Lett. 86, 131105 (2005).

${ }^{15}$ X. Meng, K. Fujita, Y. Zong, S. Murai, and K. Tanaka, Appl. Phys. Lett. 92, 201112 (2008).

${ }^{16}$ X. Meng, K. Fujita, S. Murai, and K. Tanaka, Phys. Rev. A 79, 053817 (2009).

${ }^{17}$ J. S. Lin and H. T. Chiu, J. Polym. Res. 10, 105 (2003).

${ }^{18}$ D. S. Wiersma, M. P. Van Albada, and A. Lagendijk, Rev. Sci. Instrum. 66, 5473 (1995).

${ }^{19}$ S. Etemad, R. Thompson, M. J. Andrejco, S. John, and F. C. MacKintosh, Phys. Rev. Lett. 59, 1420 (1987).

${ }^{20}$ P. E. Wolf, G. Maret, E. Akkermans, and R. Maynard, J. Phys. (France) 49, 63 (1988).

${ }^{21}$ H. C. Van De Hulst, Light Scattering by Small Particles (Wiley, New York, 1957), Chap. 1, p. 5. 